\title{
Quantitative Analysis of Cytokine mRNA Expression and Protozoan DNA Load in Theileria parva-Infected Cattle
}

\author{
Shinji YAMADA ${ }^{1)}$, Satoru KONNAI ${ }^{1}$, Saiki IMAMURA ${ }^{1)}$, Martin SIMUUNZA ${ }^{2)}$, Mwelwa CHEMBENSOFU ${ }^{2}$, \\ Amos CHOTA $^{2)}$, Andrew NAMBOTA ${ }^{2)}$, Misao ONUMA ${ }^{1)}$ and Kazuhiko OHASHI ${ }^{1) *}$ \\ ${ }^{1)}$ Department of Disease Control, Graduate School of Veterinary Medicine, Hokkaido University, Sapporo, 060-0818, Japan and \\ ${ }^{2)}$ Epidemiology Section, Disease Control Department, School of Veterinary Medicine, University of Zambia, P.O. Box 32379, Lusaka, \\ Zambia
}

(Received 22 May 2008/Accepted 6 September 2008)

\begin{abstract}
Theileria parva (T. parva) causes a highly serious bovine disease called East Coast fever (ECF), which is characterized by pyrexia, dyspnea and cachexia and is of great economic importance in African countries. We hypothesize that the clinical symptoms of ECF could be explained by a cytokine dysregulation. In this study, we investigated the relationship between $T$. parva DNA load and expression levels of cytokine mRNAs in leukocytes from experimentally infected calves by quantitative PCR. The $p 104$ gene, which encodes the $T$. parva $104 \mathrm{kDa}$ microneme-rhoptry protein, was detected in cattle blood from day 10 after $T$. parva-infected tick infestation, and the protozoan DNA load was increased together with severity of disease. The mRNA expressions of pro-inflammatory cytokines, such as interleukin (IL)- $1 \beta$ and IL-6, were up-regulated with protozoan DNA load increasing. In addition, the level of a type- 2 cytokine (IL-10) transcript was also increased during the acute phase. In contrast, the down-regulation or no detectable levels of the expression of type- 1 cytokines, such as IL- 2 and interferon (IFN)- $\gamma$ were observed in $T$. parva-infected animals. Thus, our observations indicated that high protozoan load and resulting intense inflammatory responses might be involved in the severity of clinical signs observed in T. parva-infection.

KEY WORDS: bovine, cytokine, Theileria.
\end{abstract}

Theileria parva (T. parva) is a haemoprotozoan parasite transmitted by the brown ear tick, Rhipicephalus appendiculatus (R. appendiculatus), causing East Coast fever (ECF) in cattle in several African countries [4, 7, 21]. This disease causes high mortality and morbidity, and is considered as the important restriction to the improvement of the livestock industry in Africa.

T. parva is an obligate intracellular parasite and the sporozoites infect $\mathrm{T}$ or B cells causing a severe febrile disease in cattle. After differentiation into macroschizonts in bovine lymphoid cells, infected and bystander cells undergo the massive destruction of lymphocytes in thymus, spleen or lymph nodes [17]. Some macroschizonts further differentiate into merozoites. Then, merozoites invade erythrocytes and differentiate into piroplasms, which are infective to $R$. appendiculatus ticks.

The main symptoms of ECF are pyrexia, swelling of lymph nodes, anorexia and dyspnea. Finally, lung tissues are damaged by inflammatory infiltration, leading to a severe pulmonary edema [10]. These clinical signs of ECF appear during the schizont stage of the parasite and host death can occur before the emergence of piroplasm. Therefore, acute immune-response to T. parva infection targeting at the proliferating schizonts may enhance the disease progression. In other protozoan diseases such as malaria and trypanosomasis, the clinical symptoms in acute infection are

\footnotetext{
* Correspondence to: Ohashi, K., Department of Disease Control, Graduate School of Veterinary Medicine, Hokkaido University, Sapporo 060-0818, Japan.

e-mail: okazu@vetmed.hokudai.ac.jp
}

associated with pro-inflammatory cytokines $[11,24]$. The acute inflammatory response is mediated through the increased expression of pro-inflammatory cytokines, mainly tumor necrosis factor- $\alpha$ (TNF- $\alpha$ ) and interleukin- $\beta$ (IL- $1 \beta$. These cytokines are key mediators of the responses to microbial infection and induce fever, hypotension, and the production of other cytokines, chemokines and adhesion molecules. Dysregulated activation of pro-inflammatory cytokines can lead to various inflammatory disorders [5]. In addition, experimental administration of both IL- 6 and TNF- $\alpha$ to cattle induced the systemic inflammation, which is similar clinical sign to ECF $[3,13]$. This raises the possibility that the pro-inflammatory cytokines could be involved in the severity of the disease in T. parva-infected animals. However, little is known regarding the immune response during the early phase of $T$. parva infection in vivo.

T. parva transforms bovine lymphocytes, which can proliferate continuously without any exogenous growth factors and the parasite activates the host cell signal pathways including the nuclear factor $-\kappa \mathrm{B}(\mathrm{NF}-\kappa \mathrm{B})$ and the activator protein-1 (AP-1) [6]. These transcriptional factors appear to modulate the expression of several cytokines regulating cell proliferation and anti-apoptosis in parasitized cells [6]. Through in vitro studies, cytokine profiles are well documented for their roles in affecting the proliferation of $T$. parva-transformed cell lines [1]. McKeever and co-authors [19] showed that a number of T. parva-infected cell lines exhibited a variation in the mRNA expressions of IL- $1 \beta$, IL2, IL-4 and IL-6. In addition, T. parva-infected T cell clone induced the up-regulation of IL-6, IL-10 and TNF- $\alpha$ 
mRNA, which are not expressed before the infection. However, these cytokines does not appear to modulate the cell growth of all cell lines investigated and it is not clear whether cytokines are involved in ECF pathogenesis. These previous studies prompted us to examine the involvement of cytokines in the progression of this disease. Thus, in the present study, we determined if pro-inflammatory cytokines are highly expressed during the infection with T. parva, and if these cytokines could be involved in the disease progression. We examined the relationship between protozoan DNA load and acute immuno-responses in cattle infected with $T$. parva, by using the quantitative real-time PCR assay targeting the $T$. parva $104 \mathrm{kDa}$ membrane antigen (p104) gene and several other cytokine genes.

\section{MATERIALS AND METHODS}

Generation of T. parva-infected adult R. appendiculatus ticks: T. parva-infected $R$. appendiculatus ticks were generated as previously described [15]. Briefly, a calf was inoculated with $5 \mathrm{ml}$ of a 1:50 dilution of the T. parva Muguga sporozoite stabilate (kindly donated by Center for Ticks and Tick borne Diseases, Malawi). The nymphs were applied onto the calves on day 14 post infection to coincide with the onset of piroplasm parasitaemia. Engorged nymphs were collected from 5 to 8 days post tick challenge and incubated at $20^{\circ} \mathrm{C}$, with $95 \%$ relative humidity, for 6 weeks during which time they molted into adults. The T. parva-infected ticks were used for experimental infection of cattle. The infection rate of adult $R$. appendiculatus ticks was determined by $T$. parva-specific nested PCR described previously [16].

Experimental infection: Four Friesian calves (1-year old) were infested with $T$. parva-infected $R$. appendiculatus ticks generated as described above. Calves were maintained under a strict acaricidal control regime at Experimental Animal Facility, School of Veterinary Medicine, University of Zambia (UNZA). Twenty adult ticks (10 males and 10 females) from the batch described above were applied onto calf's ears with the help of ear bags and adhesive tape. Blood samples were collected starting from day 0 post tick challenge for the extraction of DNA and RNA and for microscopic examination of Giemsa-stained smear. On day 8 and 11 , needle biopsy of the parotid lymph node was performed for the microscopic examination of schizont. The clinical signs and rectal temperature were recorded on a daily basis. The cattle were treated with oxytetracyclines on day 16 post tick challenge.

DNA extraction: $T$. parva DNA were obtained from 0.5 $\mathrm{m} l$ whole blood samples using the Wizard ${ }^{\mathrm{TM}}$ Genomic DNA Purification kit (Promega, Madison, WI, U.S.A.) according to the manufacturer's instructions. DNA was extracted from blood samples collected from day 0 to day 17 post tickchallenge. The concentration of purified bovine genomic DNA was determined by measuring optical density (OD) at $260 \mathrm{~nm}$ and these DNA extracts were subjected to PCR amplification.
RNA extraction and cDNA synthesis: Total RNA was extracted from cattle leukocytes separated from $0.5 \mathrm{ml}$ whole blood samples using the TRIzol reagent (Invitrogen, Carisbad, CA, U.S.A.) according to the manufacturer's instructions. RNA samples were extracted at day $0,10,12$, 14 and 16 post tick challenge. One microgram of total RNA was treated with DNase I (Invitrogen) to remove residual DNA, and cDNA was synthesized from the total RNA with moloney murine leukemia virus (M-MLV) reverse transcriptase (TAKARA, Otsu, Japan) using oligo(dT) ${ }_{18}$ primer (Hokkaido System Science, Sapporo, Japan). The quality and quantity of RNA was determined by measuring OD at $260 \mathrm{~nm}$ and agarose gel electrophoresis.

Real-time quantitative-PCR for the detection of T. parva p104 gene: Real-time PCR amplification of the 104 gene was performed in a Light Cycler ${ }^{\mathrm{TM}}$ (Roche Diagnostics, Mannheim, Germany) as described previously [16]. A three microliter aliquot containing $100 \mathrm{ng}$ of DNA derived from $0.5 \mathrm{~m} l$ of bovine whole blood was added to a $17 \mu l$ reaction mixture containing SYBR Premix Ex Taq ${ }^{\mathrm{TM}}$ II (TAKARA) and each of the oligonucleotide primers at the final concentration of $0.2 \mu \mathrm{M}$. The primers used in this study were $T$. parva 104 primers IL4234; 5'-GGC CAA GGT CTC CTT CAG AAT ACG-3' and IL3232; 5'-TGG GTG TGT TTC CTC GTC ATC TGC-3', which produce a 277-bp fragment. It is reported that PCR primers based on p104 gene specifically amplify $T$. parva DNA, but DNA of $T$. annulata, $T$. buffeli, T. lestoqoardi, T. mutans and T. taurotragi was not amplified using the p104 primers. Furthermore, p104 is encoded by a single-copy gene conserved among different T. parva stocks [25]. Cycling condition included an initial heat denaturation at $95^{\circ} \mathrm{C}$ for $10 \mathrm{sec}$, followed by 40 cycles of $5 \mathrm{sec}$ at $95^{\circ} \mathrm{C}, 15 \mathrm{sec}$ at $65^{\circ} \mathrm{C}$ and $15 \mathrm{sec}$ at $72^{\circ} \mathrm{C}$. A serial dilution of plasmid $\left(10^{2}-10^{-4} \mathrm{pg}\right)$ encoding $T$. parva p104 gene was used to generate a standard curve for the quantification. The copy numbers were calculated by the following equation: Copy numbers $=\left(6.02 \times 10^{23}\right) \times($ plasmid concentration $\mathrm{g}) /($ Number of bases $) \times(660 \mathrm{Da} /$ base pair $)$. Each amplification procedure was done in triplicate and the mean value was calculated. The specificity of the amplification was confirmed by melting point analysis and by confirming the length of PCR products by electrophoresis.

Real-time RT-PCR for the quantitative determination of bovine cytokine message: The mRNAs investigated for transcriptional differences were those encoding IL- $1 \beta$, IL-2, IL6 , IL-10, TNF- $\alpha$ and IFN- $\gamma$. The bovine $\beta$-actin gene was amplified in each samples to monitor the concentrations of template mRNA. The primer sequences used for the amplification are shown in Table 1. Two microliters of cDNA were added to an $18 \mu l$ reaction mixture containing SYBR Premix Ex Taq ${ }^{\mathrm{TM}}$ II (TAKARA) and each of the oligonucleotide primers at the final concentration of $0.2 \mu \mathrm{M}$. Cycling condition included an initial heat denaturation at $95^{\circ} \mathrm{C}$ for $10 \mathrm{sec}$, followed by 40 cycles of $5 \mathrm{sec}$ at $95^{\circ} \mathrm{C}, 15 \mathrm{sec}$ at each annealing temperature and $15 \mathrm{sec}$ at $72^{\circ} \mathrm{C}$. Serial dilutions of plasmid encoding each cytokine or $\beta$-actin gene $\left(10^{2}\right.$ $10^{-4} \mathrm{pg}$ ) were used to generate standard curves for the quan- 
Table 1. Primer sequences used for quantitative PCR in this study

\begin{tabular}{|c|c|c|c|}
\hline Primers/Sequences $5^{\prime} \rightarrow 3^{\prime}$ & Annealing temperature $\left({ }^{\circ} \mathrm{C}\right)$ & $\begin{array}{c}\text { Size of } \\
\text { amplified } \\
\text { fragment (bps) }\end{array}$ & Reference \\
\hline \multicolumn{4}{|l|}{ IL- $1 \beta$} \\
\hline $\begin{array}{l}\text { Forward / ACCTTCATTGCCCAGGTTTCT } \\
\text { Reverse / CTGTTTAGGGTCATCAGCCTCAA }\end{array}$ & 57 & 120 & 18 \\
\hline \multicolumn{4}{|l|}{ IL-2 } \\
\hline $\begin{array}{l}\text { Forward / TTTTACGTGCCCAAGGTTAA } \\
\text { Reverse / CGTTTACTGTTGCATCATCA }\end{array}$ & 52 & 217 & 14 \\
\hline $\begin{array}{l}\text { IL-6 } \\
\text { Forward / TGAGTGTGAAAGCAGCAAGGA } \\
\text { Reverse / TACTCCAGAAGACCAGCAGTGG }\end{array}$ & 54 & 137 & 18 \\
\hline $\begin{array}{l}\text { IL-10 } \\
\text { Forward / TGCTGGATGACTTTAAGGG } \\
\text { Reverse / AGGGCAGAAAGCGATGACA }\end{array}$ & 55 & 186 & 14 \\
\hline $\begin{array}{l}\text { TNF- } \alpha \\
\text { Forward /TAACAAGCCGGTAGCCCACG } \\
\text { Reverse / GCAAGGGCTCTTGATGGCAGA }\end{array}$ & 60 & 277 & 23 \\
\hline $\begin{array}{l}\text { IFN- } \gamma \\
\text { Forward / ATAACCAGGTCATTCAAAGG } \\
\text { Reverse / ATTCTGACTTCTCTTCCGCT }\end{array}$ & 52 & 218 & 14 \\
\hline $\begin{array}{l}\beta \text {-actin } \\
\text { Forward / CGCACCACTGGCATTGTCAT } \\
\text { Reverse / TCCAAGGCGACGTAGCAGAG }\end{array}$ & 60 & 227 & 14 \\
\hline
\end{tabular}

tification. Each amplification procedure was done in triplicate and results of cytokine mRNA expression are presented as a ratio, obtained by dividing the concentration of the cytokine mRNA by that of the $\beta$-actin mRNA. The specificity of amplification was confirmed by melting point analysis and by confirming the length of PCR products by electrophoresis.

\section{RESULTS}

Protozoan DNA load of T.parva in experimentally infected cattle: In order to determine the infection rate of $T$. parva in the $R$. appendiculatus adult ticks, total DNA was extracted from 136 (68 males and 68 females) molted ticks randomly selected and analyzed for the presence of $T$. parva DNA by nested PCR. Overall, $75.7 \%$ (103/136) of ticks placed on the calf acquired T. parva infection. There was no difference in the infection rate of $T$. parva between male $(70.6 \%)$ and female $(80.9 \%)$ ticks used in this study.

Twenty ticks infected with T. parva were allowed to feed to repletion on naive cattle. After tick challenge, all of the female ticks attached to cattle ears within 1 day and engorgement of female ticks started on day 7 and finished on day 11. All tick-infested cattle developed clinical signs of ECF displaying enlarged cervical lymph nodes and dyspnoea. Rectal temperature began to rise at day 10 post tick infestation in all of the calves and continued to rise with severity of clinical signs increasing (Fig. 1).

The presence of $T$. parva schizont was confirmed in the parotid lymph node on day 11 post tick challenge in all 4 calves by microscopic examination. Piroplasms were also detected in erythrocytes by day 16 post tick challenge (Fig. $1 \mathrm{~A}-\mathrm{C})$, while no piroplasm was detected in one of the calves by the microscopic examination of Giemsa-stained smear (Fig. 1D). The quantitative PCR used in this study revealed a detection limit of $10^{-4} \mathrm{pg}(27.7$ copies $) / 100 \mathrm{ng}$ schizontinfected leukocytes DNA. T. parva infection was confirmed by real-time PCR as early as day 10 post tick challenge in blood samples. Furthermore, the T. parva DNA load was increased in accordance with the onset of ECF clinical signs in the acute stage of infection (Fig. 1). The clinical signs became severe, and rectal temperatures were over $40^{\circ} \mathrm{C}$ from day 12 post tick challenge, during which time the total $T$. parva DNA load exceeded $10^{-1} \mathrm{pg}$. One of the calves developed pulmonary edema and pyrexia $\left(41.5^{\circ} \mathrm{C}\right)$ and died on day 16 post tick challenge when total T. parva DNA load reached $10 \mathrm{pg}$, which corresponds to $2.8 \times 10^{6}$ copies $/ 100 \mathrm{ng}$ schizont-infected leukocytes DNA (Fig. 1A).

Cytokine mRNA expression in the acute phase of T.parvainfection: In order to investigate the immunological factors affecting ECF progression at early phase of the infection, we measured the mRNA expression levels of pro-inflammatory cytokines (IL-1 $\beta$, IL-6 and TNF- $\alpha$ ), IL-2, IL-10 and IFN- $\gamma$ in experimentally infected calves. The calves developed severe clinical signs and body temperatures were over $40^{\circ} \mathrm{C}$ from day 12 post tick challenge, when the total $T$. parva DNA load exceeded $10^{-2} \mathrm{pg}$. Some animals displayed the up-regulation of TNF- $\alpha$ and IL-10 mRNA during the period, though individual response varied among animals. Conversely, IL-2 and IFN- $\gamma$ were down-regulated from 10 days post tick challenge (Fig. 2). The increase in T. parva DNA load coincided with the increase in severity of the disease from days 12 to 16 post tick exposures, and this coincided with the up-regulated expressions of IL- $1 \beta$ and IL- 6 mRNAs with 2 - to 10 -fold and 2 - to 80 -fold increasing in the expressions of IL- $1 \beta$ and IL-6, respectively (Fig. 3). 

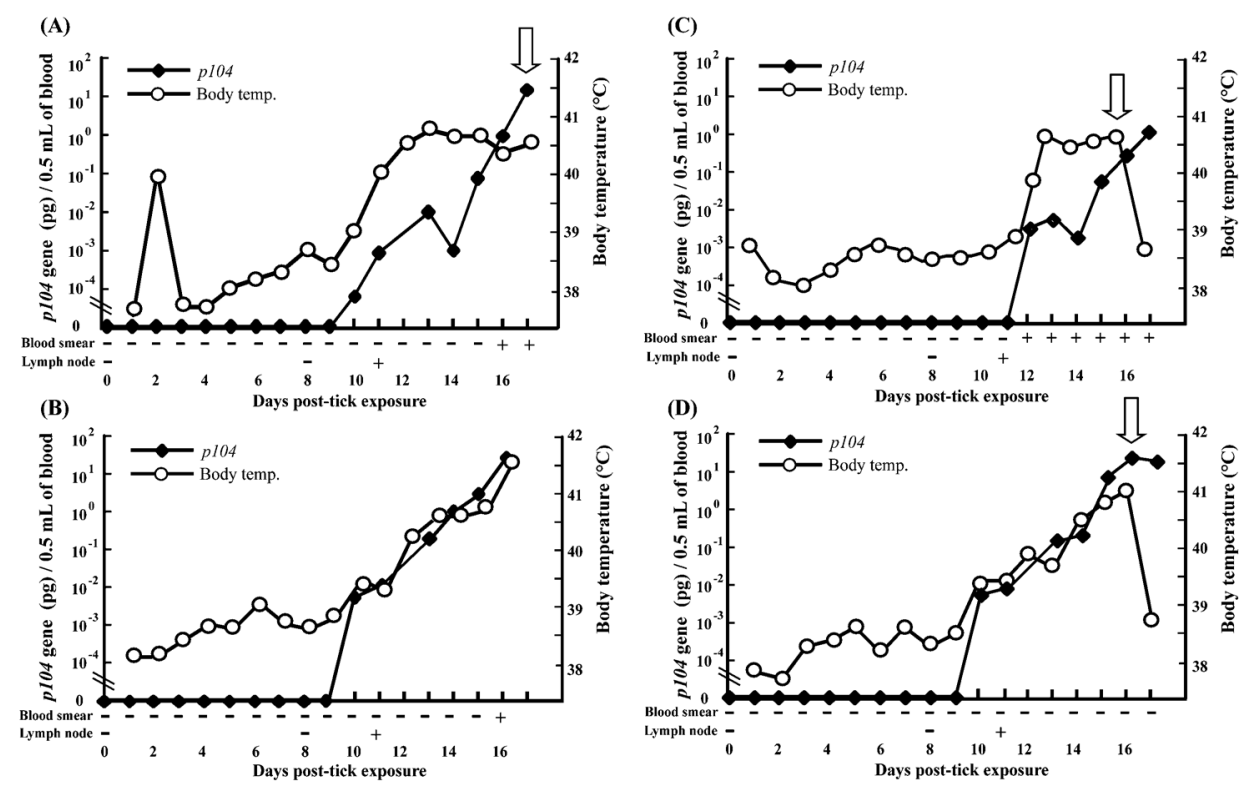

Fig. 1. Monitoring of the T. parva DNA (p104 gene) in experimentally infected calves. (A-D) Four calves (Cattle 1-4) exposed with T. parva-infected adult ticks. Each line represents T. parva DNA load (full circle) and body temperature (empty circle). Arrows indicate the treatments with oxytetracyclines. Plus $(+)$ or minus $(-)$ on the $\mathrm{X}$-axis indicates the results of piroplasm detection in blood stream or schizont in parotid lymph node by microscopic examination (+: positive, --negative).
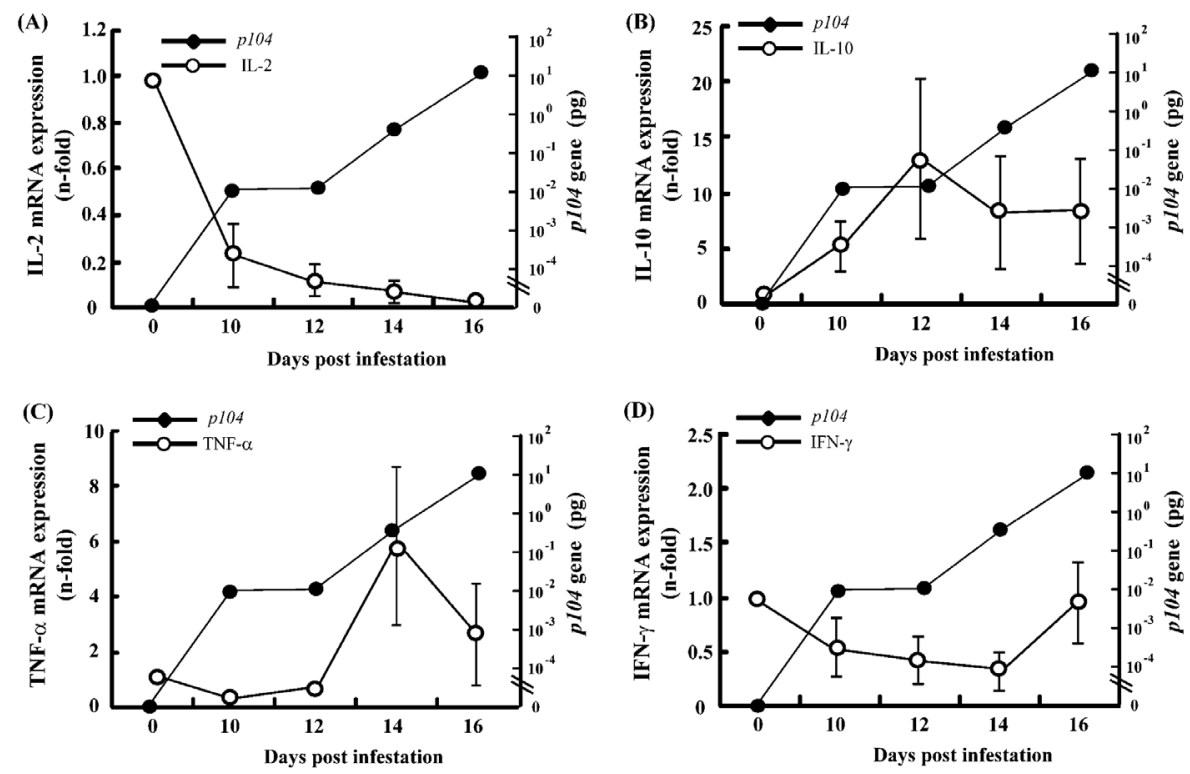

Fig. 2. Cytokine (IL-2, IL-10, TNF- $\alpha$ and IFN- $\gamma$ ) mRNA expression in experimentally infected calves. The results (A-D) are shown as fold change in mRNA levels relative to the levels of expression at the start of tick challenge (Day 0). The fold change of (A) IL-2, (B) IL-10, (C) TNF- $\alpha$ and (D) IFN- $\gamma$ mRNA is expressed as mean $\pm \mathrm{SE}$ of 4 calves exposed with $T$. parva-infected adult ticks.

\section{DISCUSSION}

There is little information on specific cytokine responses in vivo, although the roles of several cytokines in T. parvainfected cells are well documented in vitro [6]. In the present study, using an experimental model, we showed that the elevation of pro-inflammatory cytokines, IL- $1 \beta$ and IL6 , account in part for the clinical signs of ECF.

Naïve cattle were challenged with $T$. parva-infected adult $R$. appendiculatus ticks that successfully transmitted the 


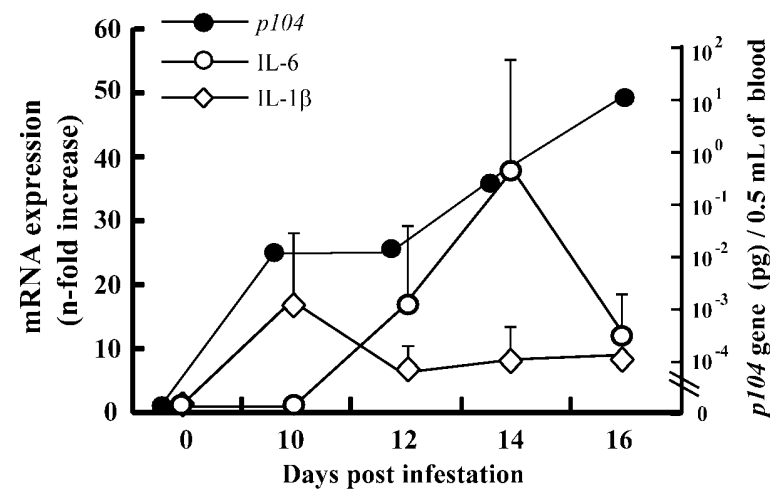

Fig. 3. Relative quantification of IL- $1 \beta$ and IL-6 mRNA, and $T$. parva DNA load. The relative increase of pro-inflammatory cytokines was observed coincided with the protozoan DNA load in four experimentally infected calves. Each line represents $T$. parva DNA load (full circle), relative change of IL-1 $\beta$ (open diamond) and IL-6 (open circle). The data is shown as mean \pm SE of 4 calves.

parasite to emulate natural infection and caused ECF. The T. parva p104 gene was detected in blood samples from all 4 calves at 12 days after tick challenge. The parasite DNA load was increased exponentially in blood stream of the cattle, and the total T. parva DNA load reached 1-10 pg/100 $n g$. Although the parasite factors that trigger the inflammatory responses are unclear, the proliferation of parasites in cattle blood was correlated with the rectal temperature as shown in Fig. 1. In this experimental infection, IL-2 and IFN- $\gamma$ mRNAs were not induced during the acute phase of the disease. It has been revealed that the proliferation of schizont-infected cells is independent of IL-2, although several cell lines appeared to be controlled by the IL-2/IL-2R autocrine loop [6]. Although we did not evaluate the number of lymphocytes in blood circulation and mRNA levels are not always indicative of protein and activity, IL-2 was not associated with the proliferation of schizont in vivo similar to the situation in vitro. The expression of IL-10 mRNA was shown to be up-regulated as protozoan DNA load was increased. As mentioned above, IL-10 has been reported to be expressed in all T. parva-transformed cell lines examined [19]. The role of IL-10 in the pathogenesis of the disease is unclear, but IL-10 may be induced as a part of a homeostatic network that protects tissues from collateral damage by excessive inflammation [20].

The up-regulated expressions of pro-inflammatory cytokines, IL- $1 \beta$ and IL-6, were accompanied with an increased proliferation of $T$. parva in vivo as shown in Fig. 3. IL-1 $\beta$ mRNA expression was observed when the $T$. parva p104 gene was detectable in blood stream by real-time PCR. The expression level of IL- $1 \beta$ mRNA peaked after day 10 post tick challenge and IL- $\beta$ mRNA was constitutively expressed in the acute phase. Expression of IL-6 was induced as DNA load of T. parva was increased, and its mRNA level was highest at day 14 post tick challenge. Among the individual cattle, there was a distinct difference in the expression pattern of pro-inflammatory cytokine mRNAs. One hypothesis is that differences in genetic background could elicit different immune responses. Nucleotide sequence polymorphisms in genes of some cytokines have been shown to determine the specificity of an individua's immune responses.

In the previous studies, a $\mathrm{T}$ cell clone infected with $T$. parva appeared to express higher cytokine mRNAs (IL-6 and TNF- $\alpha$ ) than an infected B cell clone [19], and T cells infected with $T$. parva stimulated autologous cells more efficiently than infected B cells [9]. In addition, the inoculation of cattle with $T$. parva-infected B cells has been shown to cause a mild to modest disease [22]. These results suggest that both the types of infected cells and related cytokines could be associated with pathogenesis of ECF. Recently, it has been reported that the cytokine storms in humans are strongly associated with high mortality and increased severity of disease in several fatal infections such as malaria [8], severe acute respiratory syndrome [12], Ebola virus infection [2] and highly pathogenic avian influenza [26]. Among these diseases, main causative cytokines for the cytokine storm seem to be pro-inflammatory cytokines such as IL- $1 \beta$ IL- 6 and TNF- $\alpha$. In the case of malaria, expressions of pro-inflammatory cytokines are increased with increased severity of disease resulting in disseminated intravascular coagulation. These observations raise the possibility that protozoa-induced cytokine dysregulation as seen in this study may contribute to disease severity of ECF.

In conclusion, we report that certain pro-inflammatory cytokine transcripts from T. parva-infected cattle are highly expressed during the acute phase when the parasite is proliferating. These cytokines form a part of the acute-phase response in cattle. The examination of cytokine profiles in T. parva-infected cattle would help the understanding of the disease pathogenesis. The elucidation of the mechanism of the pathogenesis of ECF may help developing methods needed to control the disease.

ACKNOWLEDGEMENT. We deeply thank Dr. Itabajara da Silva Vaz Junior (Centro de Biotecnologia, Universidade Federal do Rio Grande do Sul) for his critical reading of the manuscript.

\section{REFERENCES}

1. Ahmed, J.S., Schnittger, L. and Mehlhorn, H. 1999. Theileria schizonts induce fundamental alterations in their host cells. Parasitol. Res. 85: 527-538.

2. Baize, S., Leroy, E.M., Georges, A.J., Georges-Courbot, M.C., Capron, M., Bedjabaga, I., Lansoud-Soukate, J. and Mavoungou, E. 2002. Inflammatory responses in Ebola virus-infected patients. Clin. Exp. Immunol. 128: 163-168.

3. Bielefeldt Ohmann, H., Campos, M., Snider, M., Raipin, N., Beshkorwayne, T., Popowych, Y., Lawman, M.J., Rossi, A. and Babiuik, L.A. 1989. Effect of chronic administration of recombinant bovine tumour necrosis factor to cattle. Vet. Pathol. 26: 462-472.

4. Billiouw, M., Vercruysse, J., Marcotty, T., Speybroeck, N., 
Chaka, G. and Berkvens, D. 2002. Theileria parva epidemics: a case study in eastern Zambia. Vet. Parasitol. 107: 51-63.

5. Dinarello, C.A. 1996. Biological basis for interleukin-1 in disease. Blood 87: 2095-2147.

6. Dobbelaere, D. and Heussler, V. 1999. Transformation of leukocytes by Theileria parva and T. annulata. Annu. Rev. Microbiol. 53: 1-42.

7. Geysen, D., Bishop, R., Skilton, R., Dolan, T.T. and Morzaria, S.P. 1999. Molecular epidemiology of Theileria parva infections in the field. Trop. Med. Int. Health 4: 21-27.

8. Ghosh, K. and Shetty, S. 2008. Blood coagulation in falciparum malaria-a review. Parasitol. Res. 102: 571-576.

9. Goddeeris, B.M. and Morrison, W.I. 1987. The bovine autologous Theileria mixed lymphocyte reaction: influence of monocytes and phenotype of the parasitized stimulator cell on proliferation and parasite specificity. Immunology 60: 63-69.

10. Gwamaka, M., Matovelo, J.A., Mtambo, M.M., Mbassa, G.K., Maselle, R.M. and Boniphace, S. 2004 The effect of dexamethasone and promethazine in combination with buparvaquone in the management of East Coast fever. Onderstepoort J. Vet. Res. 71: 119-128.

11. Hirunpetcharat, C., Finkelman, F., Clark, I.A. and Good, M.F. 1999. Malaria parasite-specific Th1-like T cells simultaneously reduce parasitaemia and promote disease. Parasite Immunol. 21: $319-329$.

12. Huang, K.J., Su, I.J., Theron, M., Wu, Y.C., Lai, S.K., Liu, C.C. and Lei, H.Y. 2005. An interferon-gamma-related cytokine storm in SARS patients. J. Med. Virol. 75: 185-194.

13. Jongen-Lavrencic, M., Peeters, H.R.M., Rozemuller, H., Rombouts, W.J.C., Martens, A.C.M., Vreugdenhil, G., Pillay, M., Cox, P.H., Bijser, M., Brutel, G., Breedveld, F.C. and Swaak, A.J.G. 1996. IL-6-induced anaemia in rats: possible pathogenic implications for anaemia observed in chronic inflammations. Clin. Exp. Immunol. 103: 328-334.

14. Konnai, S., Usui, T., Ohashi, K. and Onuma, M. 2003. The rapid quantitative analysis of bovine cytokine genes by realtime RT-PCR. Vet. Microbiol. 94: 283-294.

15. Konnai, S., Yamada, S., Imamura, S., Simuunza, M., Chembensof, M., Chota, A., Nambota, A., Ohashi, K. and Onuma, M. 2007. Attachment duration required for Rhipicephalus appendiculatus to transmit Theileria parva to the host. Vector Borne Zoonotic Dis. 7: 241-248.

16. Konnai, S., Imamura, S., Nakajima, C., Witola, W.H., Yamada, S., Simuunza, M., Nambota, A., Yasuda, J., Ohashi, K. and Onuma, M. 2006. Acquisition and transmission of Theileria parva by vector tick, Rhipicephalus appendiculatus. Acta Trop.
99: $34-41$.

17. Mbassa, G.K., Balemba, O., Maselle, R.M. and Mwaga, N.V. 1994. Severe anemia due to haematoepoietic cell destruction in field cases of East Coast fever in Tanzania. Vet. Parasitol. 52: 243-256.

18. McGuire, K., Manuja, A., Russell, G.C., Springbett, A., Craigmile, S.C., Nichani, A.K., Malhotrab, D.V. and Glass, E.J. 2004. Quantitative analysis of pro-inflammatory cytokine mRNA expression in Theileria annulata-infected cell lines derived from resistant and susceptible cattle. Vet. Immunol. Immunopathol. 99: 87-98.

19. McKeever, D.J., Nyanjui, K. and Ballingall, K. 1997. In vitro infection with Theileria parva is associated with IL10 expression in all bovine lymphocyte lineages. Parasite Immunol. 19: 319-324.

20. Mege, J.L., Meghari, S., Honstettre, A., Capo, C. and Raoult, D. 2006. The two faces of interleukin 10 in human infectious diseases. Lancet Infect. Dis. 6: 557-569.

21. Minjauw, B., Otte, M.J., James, A.D., de Castro, J.J., Permin, A. and Di Giulo, G. 1998. An outbreak of East Coast fever in a herd of Sanga cattle in Lutale, Central Province of Zambia. Prev. Vet. Med. 35: 143-147.

22. Morrison, W. I., MacHugh, N.D. and Lalor, P.A. 1996. Pathogenicity of Theileria parva is influenced by the host cell type infected by the parasite. Infect. Immun. 64: 557-562.

23. Neuvians, T.P., Schams, D., Berisha, B. and Pfaffl, M.W. 2004. Involvement of proinflammatory cytokines, mediators of inflammation, and basic fibroblast growth factor in prostaglandin F2alpha-induced luteolysis in bovine corpus luteum. Biol. Reprod. 70: 473-480.

24. Sileghem, M., Flynn, J.N., Logan-Henfrey, L. and Ellis, J. 1994. Tumour necrosis factor production by monocytes from cattle infected with Trypanosoma (Duttonella) vivax and Trypanosoma (Nannomonas) congolense: possible association with severity of anaemia associated with disease. Parasite Immunol. 16: 51-54.

25. Skilton, R.A., Bishop, R.P., Katende, J.M., Mwaura, S. and Morzaria, S.P. 2002. The persistence of Theileria parva infection in cattle immunized using two stocks which differ in their ability to induce a carrier state: analysis using a novel blood spot PCR assay. Parasitology 124: 265-276.

26. To, K.F., Chan, P.K., Chan, K.F., Lee, W.K., Lam, W.Y. and Wong, K.F. 2001. Pathology of fatal human infection associated with avian influenza A H5N1 virus. J. Med. Virol. 63: 242-246. 